\title{
Histone deacetylase inhibitors induce attenuation of Wnt signaling and TCF7L2 depletion in colorectal carcinoma cells
}

\author{
SILKE GÖTZE ${ }^{1}$, MONIQUE COERSMEYER ${ }^{2}$, OLIVER MÜLLER ${ }^{3}$ and SONJA SIEVERS ${ }^{1}$ \\ ${ }^{1}$ Max Planck Institute of Molecular Physiology, Dortmund, Germany; ${ }^{2}$ Swiss Institute for Experimental Cancer Research, \\ Lausanne, Switzerland; ${ }^{3}$ University of Applied Sciences, Kaiserslautern, Germany
}

Received April 21, 2014; Accepted May 30, 2014

DOI: 10.3892/ijo.2014.2550

\begin{abstract}
Histone deacetylase inhibitors (HDIs) specifically affect cancer cells by inducing cell cycle arrest, activate apoptotic pathways and re-activate epigenetically silenced tumor suppressor genes, but their pleiotropic mode of action is not fully understood. Despite the clinical effects of HDIs in the treatment of hematological malignancies, their potency against solid tumors is still unclear. We investigated the effects and mechanisms of HDI action in colorectal carcinoma cell lines with an activated Wnt signaling pathway, which is implicated in different aspects of tumorigenesis, including cell proliferation, apoptosis, angiogenesis and metastasis. We assessed the effects of HDI treatment in colorectal carcinoma cell lines by measuring histone hyperacetylation, cell viability and expression of Wnt target genes. Upon treatment with HDIs of the hydroxamate class, we found attenuation of Wnt signaling with concomitant induction of apoptosis and colorectal cancer cell death. Strikingly, the effects of HDIs on Wnt signaling were independent of histone hyperacetylation, thus we investigated the role of non-histone target proteins of histone deacetylases (HDACs). The compounds TSA and SAHA induced a rapid proteasome-dependent depletion of the Wnt transcription factor TCF7L2, which may be mediated by inhibition of HDAC 6 and 10. Our findings provide a molecular rationale for the use of HDIs against colorectal carcinomas with activated Wnt signaling.
\end{abstract}

\section{Introduction}

Histone deacetylase inhibitors (HDIs) unfold specific effects against cancer cells. Their pleiotropic mode of action is not

Correspondence to: Dr Sonja Sievers, Max Planck Institute of Molecular Physiology, Otto-Hahn-Strasse 11, D-44227 Dortmund, Germany

E-mail: sonja.sievers@mpi-dortmund.mpg.de

Key words: histone deacetylase inhibitors, Wnt signaling, TCF7L2, colon carcinoma fully understood but includes induction of cell cycle arrest, direct activation of apoptotic pathways and reactivation of epigenetically silenced tumor suppressor genes $(1,2)$. However, many genes are downregulated by HDI treatment (3). Often, this downregulation is mediated via many non-histone target proteins of histone deacetylases (HDACs) (4) such as TP53 or RUNX3 $(5,6)$.

Despite the high clinical potential of HDIs against hematological malignancies, their effectiveness against solid tumors has not been proven yet $(7,8)$. Different HDIs were shown to induce CDKN1A (encoding the cell cycle kinase inhibitor p21) expression and cell death in colorectal carcinoma cell lines $(9,10)$. In a mouse model of colorectal cancer, inhibition of HDAC2 led to a significant reduction in adenoma formation (11). Yet, clinically proven benefits of HDI treatment are observed in individual cases only (7). However, solid cancer types in clinical studies are diverse and so are the genetic aberrations in these tumors. Hence, we sought to analyze HDI's properties in one specific type of cancer cells, i.e., colorectal carcinoma cell lines characterized by constitutively active Wnt signaling.

Wnt signaling controls many cellular processes during neoplastic transformation (12) and thus represents an important therapeutic target. In a Wnt activated cell, the protein $\beta$-catenin, the central player of the pathway, accumulates in the cytoplasm and enters the nucleus. Nuclear $\beta$-catenin associates with transcription factors of the TCF/LEF family and recruits transcriptional co-activators and chromatin remodeling complexes which in concert drive the expression of target genes (13). Wnt target genes include CCNDI, $M M P 7, V E G F, M Y C$, survivin and also genes involved in feed-back regulation such as TCF7L2 or $L E F 1$. In many epithelial cancers, the Wnt pathway is constitutively active due to mutations in different components of the pathway as for instance almost every colorectal carcinoma harbors either an $A P C$ or a $\beta$-catenin mutation (14).

The mode of action of HDIs in respect to Wnt signaling is not well defined. HDIs were shown to downregulate the expression of single endogenous Wnt target genes, e.g., $C C N D 1, V E G F$ and survivin $(15,16)$. In other studies activation of Wnt signaling due to HDI treatment could be demonstrated (17). We assessed the effects of HDI treatment in colorectal carcinoma cell lines to further elucidate the mode of HDI action. 


\section{Materials and methods}

Cell culture and drug treatment. Colorectal carcinoma cell lines SW480 and HCT116 were obtained from the American Type Culture Collection (LGC Promochem, Wesel, Germany). Cells were maintained in Dulbecco's modified Eagle's medium (DMEM) including 10\% fetal bovine serum (FBS), sodium pyruvate, L-glutamine and non-essential amino acids (PAN Biotech, Aidenbach, Germany) at $37^{\circ} \mathrm{C}$ in a $7.5 \% \mathrm{CO}_{2}$ humidified chamber. Cells were treated with $1 \mu \mathrm{M}$ trichostatin A (TSA; Sigma, Munich, Germany) or $5 \mu \mathrm{M}$ suberoylanilide hydroxamic acid (SAHA; Cayman Chemicals, Ann Arbor, MI, USA) dissolved in ethanol and $1 \mathrm{mM}$ valproic acid (VPA; Sigma) dissolved in PBS. Vehicle treatment with either ethanol or PBS served as internal control. The proteasome inhibitors MG132 and Epoxomicin (both from Calbiochem, Darmstadt, Germany) were dissolved in DMSO.

Cellular assays. Cell growth and viability were evaluated by using bromodeoxyuridine incorporation (BrdU) and methyl thiazol tetrazolium bromide reduction (MTT) assays, respectively. Cells were plated in triplicates in 96 -well plates. After treatment with HDIs and control substances for 24,30 and $48 \mathrm{~h}$ or 24 and $48 \mathrm{~h}$ after siRNA treatment, MTT or BrdU assay (BrdU cell proliferation ELISA; Roche, Mannheim, Germany) was carried out. The mean absorbance values of HDI-treated cells were normalized to the mean absorbance values of vehicle-treated cells. To detect apoptotic cells, the Apo-One homogeneous Caspase-3/7 assay (Promega, Madison, WI, USA) was carried out according to the manufacturer's instructions.

For cell cycle analysis, cells were trypsinized after $24 \mathrm{~h}$ HDI treatment or $48 \mathrm{~h}$ after siRNA transfection. The cell cycle was measured via propidium iodide incorporation on a LSRII flow cytometer and analyzed with FACS DiVa software (Becton Dickinson, Franklin Lakes, NJ, USA). Data evaluation was performed with FlowJo software (TreeStar Inc., Ashland, OR, USA).

HDAC knockdown - siRNA transfection. HDAC knockdown was performed with siGENOME SMARTpool siRNA from Dharmacon for the genes HDAC6 (M-003499-00), HDAC10 (M-004072-00) and HDAC11 (M-004258-00). Transfection was carried out with DharmaFECT1 (Dharmacon, Lafayette CO, USA) at a ratio of 1:40 in HCT116 and 1:50 in SW480 cells according to the manufacturer's instructions. Experiments were performed 24 to $72 \mathrm{~h}$ after siRNA transfection. Specific target knockdown was measured on mRNA level by quantitative real-time PCR after 24 or $48 \mathrm{~h}$.

Reverse transcription real-time PCR. RNA isolation was carried out with the NucleoSpin RNA II kit (Macherey-Nagel, Düren, Germany), cDNA synthesis was performed with RevertAID H Minus First Strand cDNA Synthesis kit (Fermentas, St. Leon-Rot, Germany). The ABsolute QPCR SYBR-Green ROX Mix (Abgene, Epsom, UK) was used for quantitative realtime PCRs on a GeneAmp 5700 Sequence detection system (Applied Biosystems, Foster City, CA, USA). Primer sequences are stated in Table I. Real-time PCR data was analyzed using the $\Delta \Delta \mathrm{Ct}$ method whereby the relative expression change of a treated sample over a control sample is: fold change $=2^{-\Delta \Delta C \mathrm{Ct}}$.
Table I. Primer sequences.

\begin{tabular}{ll} 
Primer name & \multicolumn{1}{c}{ Sequence $5^{\prime} \rightarrow 3^{\prime}$} \\
\hline LEF1 & \\
Forward & CTTGGTGAACGAGTCTGAAATC \\
Reverse & GTGTTCTCTGGCCTTGTCGTG
\end{tabular}

TCF7L2

Forward

GGAGCCTCCAGAGTAGACAAG

Reverse

CCACTGGCACTTTGTTAGAGAC

Cyclin D1

Forward

Reverse

c-Myc

Forward

Reverse

VEGF

Forward

GCCTGAACCTGAGGAGCCCCA

GTCACACTTGATCACTCTGG

Reverse

TCCTGAGACAGATCAGCAACAACCG

TCCTCTGGCGCTCCAAGACGTT

MMP7

Forward

Reverse

CATGACAGCGCCCCTTCCTGG

TGTGAGGACATAGGTCCTTTTAGGCTG

GAPDH

Forward

TGTTGCAGAATACTCACTATTTCC

GATCCACTGTAATATGCGGTAAG

Reverse

GCTCTCCAGAACATCATCCCTGCC AGGCCATGCCAGTGAGCTTCC

Western blot analysis. Total cell lysates were made from HCT116 or SW480 cells after treatment with HDIs for 3, 6 and 16 or $20 \mathrm{~h}$. After SDS gel electrophoresis, western blot analysis was used to determine the relative protein levels of TCF7L2 and $\beta$-catenin in total cell lysates. GAPDH was used as an internal control. The antibodies were as follows: TCF7L2 (Cell Signaling Technology, Danvers, MA, USA) 1:2,500 in $5 \%$ BSA/TBST; $\beta$-catenin (BD Biosciences, Franklin Lakes, NJ, USA) 1:10,000 in 0.5\% SlimFast/TBST; GAPDH (Abcam) $1: 30,000$ in $5 \%$ milk/TBST. Band intensities were determined using AIDA software (Raytest, Straubenhardt, Germany). The band intensity (BI) of the analyzed protein was referred to the $\mathrm{BI}$ of GAPDH to give the normalized BI. The normalized BI of control treated cells was set to $100 \%$.

Immunoprecipitation. For cytosolic and nuclear fractionation of cells, HCT116 cells were grown in $10-\mathrm{cm}$ dishes and HDI treated for $16 \mathrm{~h}$. Cells were washed with ice-cold PBS, scraped into a microcentrifuge tube and centrifuged for $5 \mathrm{~min}$ at 1,600 rpm. The cell pellet was resuspended in $100 \mu \mathrm{l}$ membrane lysis buffer [10 mM HEPES, pH 8.0; $10 \mathrm{mM}$ $\mathrm{KCl} ; 1.5 \mathrm{mM} \mathrm{MgCl}_{2}$; $1 \mathrm{mM}$ DTT; $1 \mathrm{X}$ Complete Protease Inhibitor Cocktail (Roche)] and incubated for $15 \mathrm{~min}$ on ice. NP-40 was added to a final concentration of $1 \%$. After vortexing for $10 \mathrm{sec}$, the sample was centrifuged for $3 \mathrm{~min}$ at $13,000 \mathrm{rpm}$. The supernatant is the cytoplasmic fraction. The pellet containing the nuclei was washed 3 times with $500 \mu 1 \mathrm{membrane}$ lysis buffer and centrifuged for $3 \mathrm{~min}$. Then the pellet was resuspended in $100 \mu 1$ ice cold nuclear enve- 
lope lysis buffer [20 mM HEPES, pH 8.0; $1.5 \mathrm{mM} \mathrm{MgCl}$; 25\% glycerol; $0.42 \mathrm{M} \mathrm{NaCl}$; 0.2 mM EDTA; 1 mM DTT; 1X Complete Protease Inhibitor Cocktail (Roche)] and vortexed for $30 \mathrm{sec}$. After rotation at $4^{\circ} \mathrm{C}$ for $30 \mathrm{~min}$, samples were centrifuged for $15 \mathrm{~min}$ at $13,000 \mathrm{rpm}$. The protein concentration of the supernatant was determined by Bradford assay. Concentrations of HDI- and vehicle-treated samples were adjusted to $3 \mu \mathrm{g} / \mu \mathrm{l}$. Lysates were used immediately or stored in aliquots at $-80^{\circ} \mathrm{C}$ until use.

For immunoprecipitation, protein $\mathrm{G}$ coupled magnetic beads (Dynabeads, Invitrogen) were used according to the manufacturer's instructions. In brief, $50 \mu \mathrm{l}$ of beads were loaded with $5 \mu \mathrm{g}$ of antibody. After washing, the loaded beads were incubated with cellular lysates for 10 to $20 \mathrm{~min}$. After several washing steps, the beads were resuspended in 5X SDS loading buffer and heated to $70^{\circ} \mathrm{C}$ for $10 \mathrm{~min}$.

To test the acetylation status of $\beta$-catenin, immunoprecipitations were carried out with an anti- $\beta$-catenin antibody (BD Biosciences) followed by western blot analysis with an anti- $\beta$-catenin antibody or an anti-acetylated $\beta$-catenin antibody (Cell Signaling Technology).

Statistical analysis. Statistical calculations were carried out with the GraphPad QuickCalcs online calculator (www. graphpad.com/quickcalcs) and STATISTICA 8 software (StatSoft, Hamburg, Germany) using different t-tests as indicated in the text. A p-value of $<0.05$ was considered to indicate statistical significance. Significant results are indicated by ${ }^{*} \mathrm{p}<0.05 ;{ }^{* *} \mathrm{p}<0.01$ or ${ }^{* * *} \mathrm{p}<0.001$ in the figures.

\section{Results}

The HDIs SAHA and TSA attenuate proliferation and induce apoptosis in Wnt-activated colorectal carcinoma cell lines. We analyzed the effects of the HDIs SAHA, TSA and VPA on the growth of the colorectal carcinoma cell lines HCT116 and SW480. Both cell lines possess an activated Wnt signaling pathway due to mutations in $\beta$-catenin or $A P C$, respectively. SAHA and TSA belong to the chemical class of hydroxamic acids; whereas VPA is a short-chain fatty acid. Treatment with hydroxamic acids led to a strong reduction in the number of viable cells, while VPA had no, or only little effect on viability even after $48 \mathrm{~h}$ as measured by MTT assay (Fig. 1A). Furthermore, a BrdU assay revealed a pronounced decrease in the cell proliferation rate of SAHA- or TSA-treated cells as opposed to VPA-treated cells (Fig. 1B). To gain further insights into the underlying mechanisms we carried out an apoptosis detection assay. After treatment with hydroxamic acids for 24 and $30 \mathrm{~h}$, the number of apoptotic cells strongly increased (Fig. 1C). After $48 \mathrm{~h}$, the relative apoptotic rate dropped due to the low number of living cells. In contrast, VPA treatment induced no significant changes in the apoptosis rate. These findings were further underlined by flow cytometric cell cycle analysis revealing an increase in the apoptotic cell fraction and an induction of a G2/M-phase cell cycle arrest in response to TSA and SAHA (Fig. 2). In contrast, treatment with VPA led to little, or no changes in the cell cycle.

The HDIs SAHA and TSA repress Wht target genes in colorectal carcinoma cells. In order to explore how HDIs influence Wnt regulated genes we measured the expression of endogenous Wnt target genes by quantitative real-time PCR after treatment with HDIs (Fig. 3). Analyzed Wnt target genes included $M Y C, C C D N 1$ (encoding cyclin D1), VEGF, MMP7, survivin and the two transcription factors $L E F 1$ and TCF $7 L 2$ which are integral parts of the pathway. We observed downregulation of all analyzed genes upon $20 \mathrm{~h}$ treatment with hydroxamic acids whereas VPA treatment left the expression of most genes unchanged (Fig. 4). In HCT116 cells, the expression of CCDN1, MYC, VEGF, MMP7, survivin and TCF7L2 was significantly downregulated after 16 to $20 \mathrm{~h}$ exposure to SAHA or TSA, but not to VPA. In SW480 cells, the expression levels of CCDN1, MYC, survivin and $L E F 1$ were significantly downregulated by SAHA or TSA, but not by VPA treatment. Only MYC expression, which is known to be regulated by a variety of mechanisms (18), was significantly downregulated by VPA treatment in HCT116.

$\beta$-catenin acetylation status remains unchanged in response to TSA treatment. Based on recent findings that the $\beta$-catenin protein can be acetylated, we questioned if a modification of this central player of the Wnt pathway may be involved in the transcriptional downregulation of target genes in response to HDI treatment. Immunoprecipitation experiments with anti-acetylated $\beta$-catenin antibody and nuclear lysates from HCT116 cells after $16 \mathrm{~h}$ TSA treatment revealed no difference in the amount of precipitated acetylated $\beta$-catenin between TSA- and vehicle-treated cells (Fig. 5). Similarly, there was no change in the total amount of $\beta$-catenin protein in total cell lysates.

The HDIs SAHA and TSA induce TCF7L2 depletion. We investigated if TCF7L2 might be a non-histone target of HDACs by analyzing the relative TCF7L2 protein level in total HCT116 cell lysates after HDI treatment. We found a strong depletion of TCF7L2 protein after $20 \mathrm{~h}$ SAHA and TSA, but not after VPA treatment $(p<0.001)$ (Fig. 6). This TCF7L2 depletion could not be attributed to transcriptional downregulation alone, as, according to the N-end rule (19), the estimated half-life of the TCF7L2 protein is $30 \mathrm{~h}$. Hence, TCF7L2 depletion might be partly caused by proteasomal degradation. In this case, co-treatment with proteasome inhibitors should attenuate the effect of the HDI treatment. We treated HCT116 cells with TSA for $16 \mathrm{~h}$ and added one of the proteasome inhibitors Epoxomicin or MG132 4 or $6 \mathrm{~h}$ before the end of the treatment, respectively. As control we treated the cells with the proteasome inhibitors alone. Co-treatment with HDIs and proteasome inhibitors indeed attenuated the downregulation of TCF7L2 protein level (Fig. 6C and D). Treatment with proteasome inhibitors alone did not alter the TCF7L2 level substantially (Fig. 6E), which showed that TCF7L2 was not subjected to constant proteasomal degradation under normal conditions. Thus we demonstrated that HDIs of the hydroxamate class induce a strong proteasome-dependent depletion of TCF7L2.

Knockdown of single HDACs cannot mimic the HDI-induced attenuation of cell proliferation. The previous experiments showed that the hydroxamic acids SAHA and TSA, which inhibit all known HDACs, induced apoptotic cell death 
A
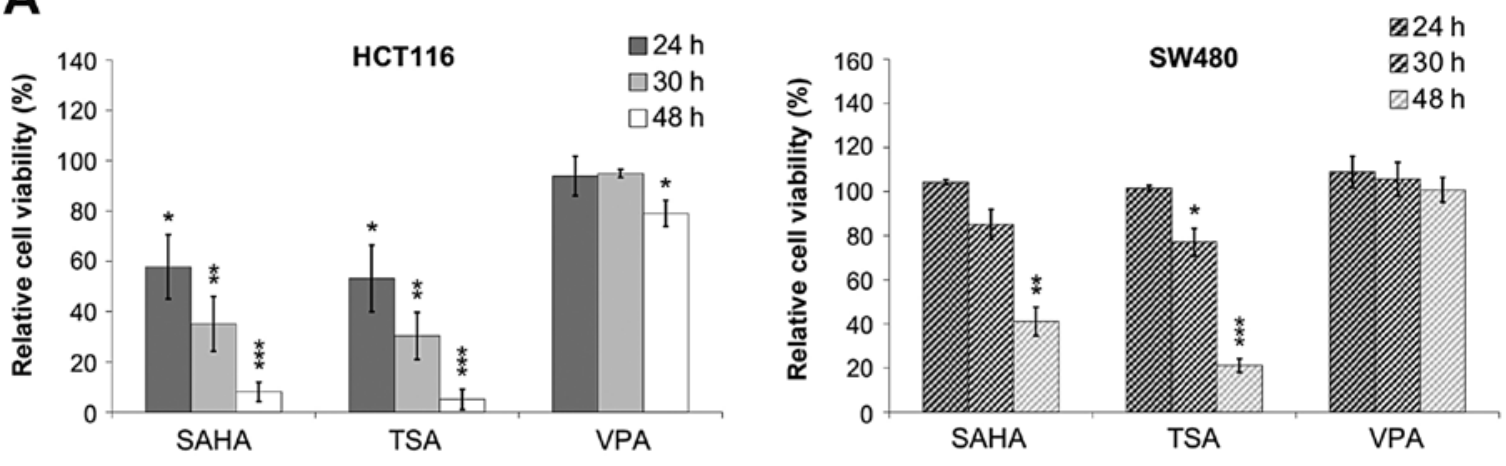

B
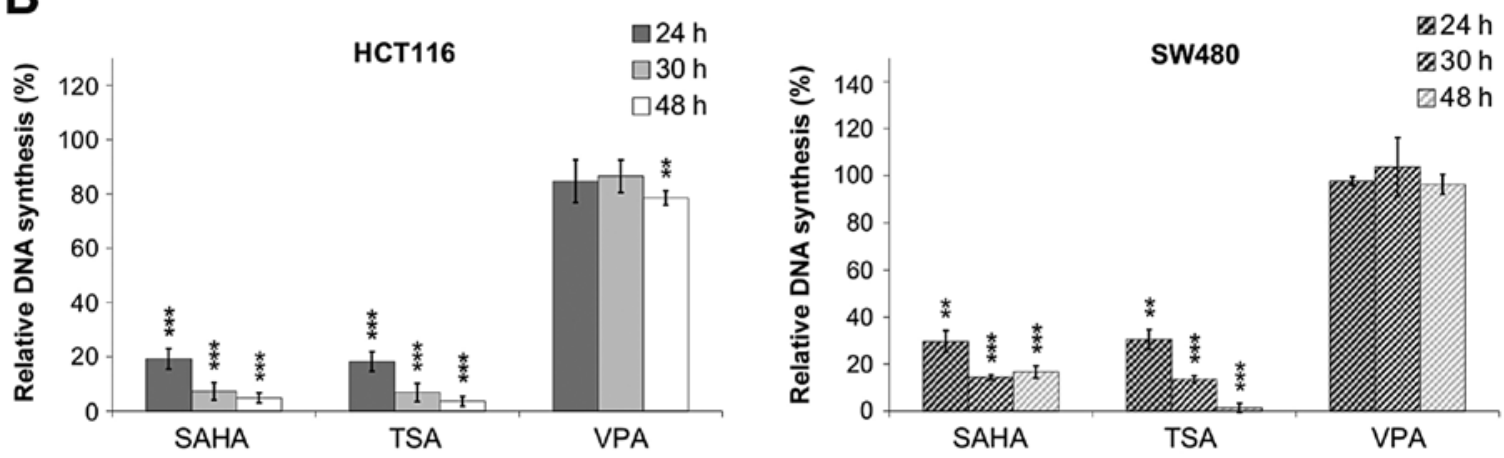

C
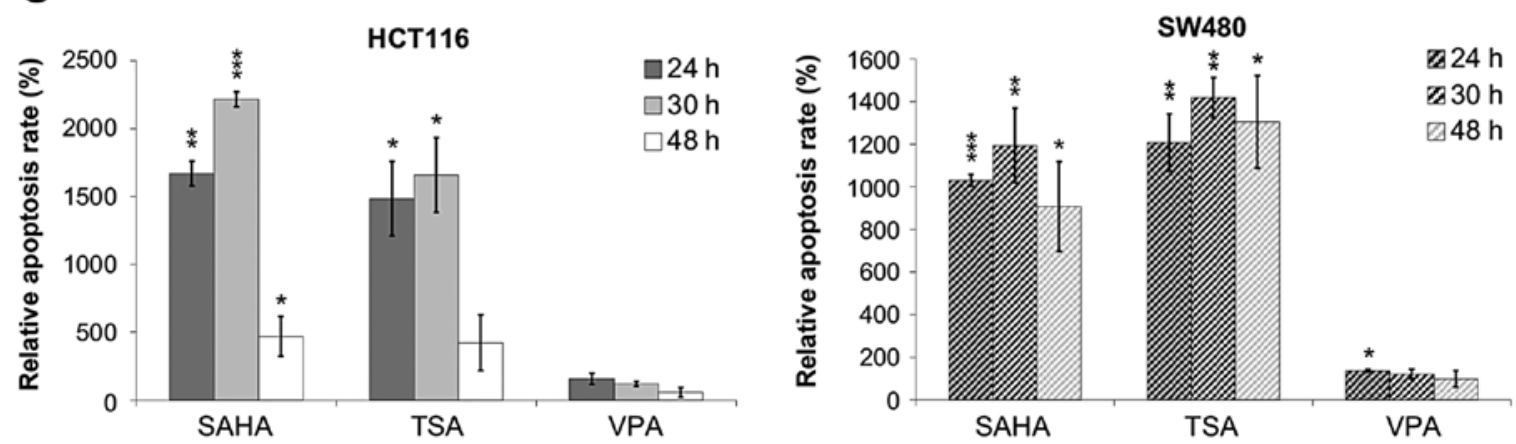

Figure 1. Effects of HDI treatment on colorectal carcinoma cell growth and apoptosis. Cells were treated with $5 \mu \mathrm{M}$ SAHA, $1 \mu \mathrm{M}$ TSA or $1 \mathrm{mM}$ VPA for the indicated time spans. The mean values of HDI-treated cells were normalized to the mean values of vehicle-treated cells, which were defined as $100 \%$. Results are means of at least three independent experiments of (A) MTT, (B) BrdU and (C) apoptosis detection assay. Significant results are indicated by ${ }^{*} \mathrm{p}<0.05 ;{ }^{* * *} \mathrm{p}<0.01$ or ${ }^{* * * *} \mathrm{p}<0.001$.
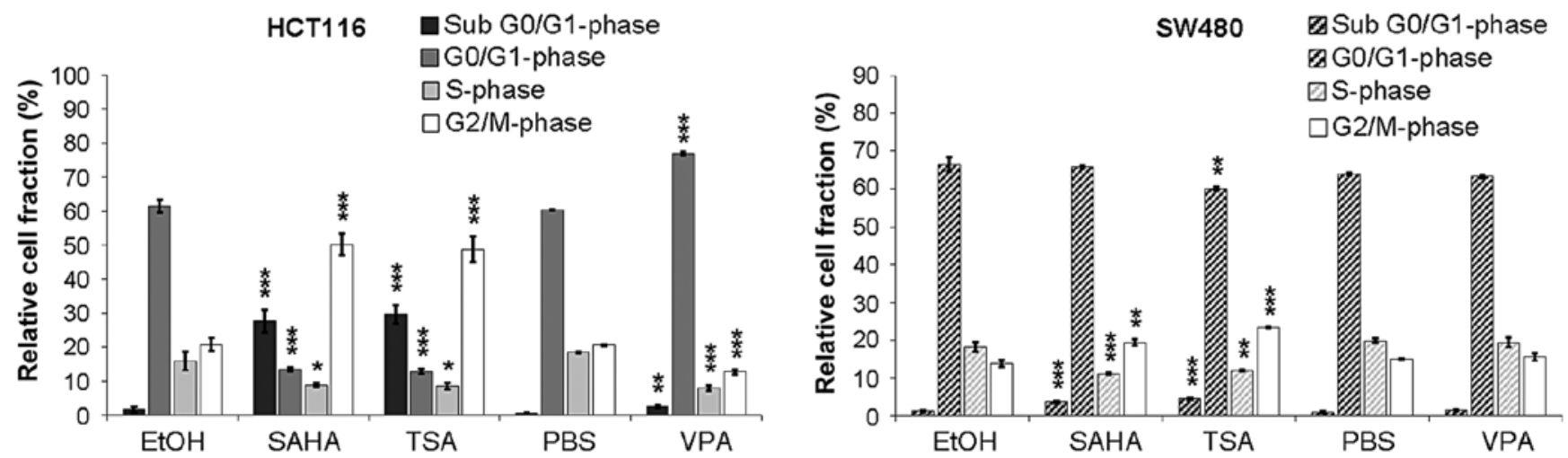

Figure 2. Effect of HDI treatment on the cell cycle. Cell cycle analysis was performed on a BD LSRII flow cytometer after propidium iodide incorporation. HCT116 and SW480 cells were treated with $5 \mu \mathrm{M}$ SAHA, $1 \mu \mathrm{M} \mathrm{TSA}, 1 \mathrm{mM}$ VPA or the buffer substances EtOH or PBS for $24 \mathrm{~h}$ before measurement. The diagram shows the relative fraction of cells in the different cell cycle phases. Results are means of three independent experiments. Interestingly SAHA and TSA treatment led to an increase in the G2/M-phase, while VPA induced a cell cycle arrest in G0/G1-phase in HCT116 cells only. Significant results are indicated by ${ }^{*} \mathrm{p}<0.05$; *** $\mathrm{p}<0.01$ or ${ }^{* * *} \mathrm{p}<0.001$. 
A
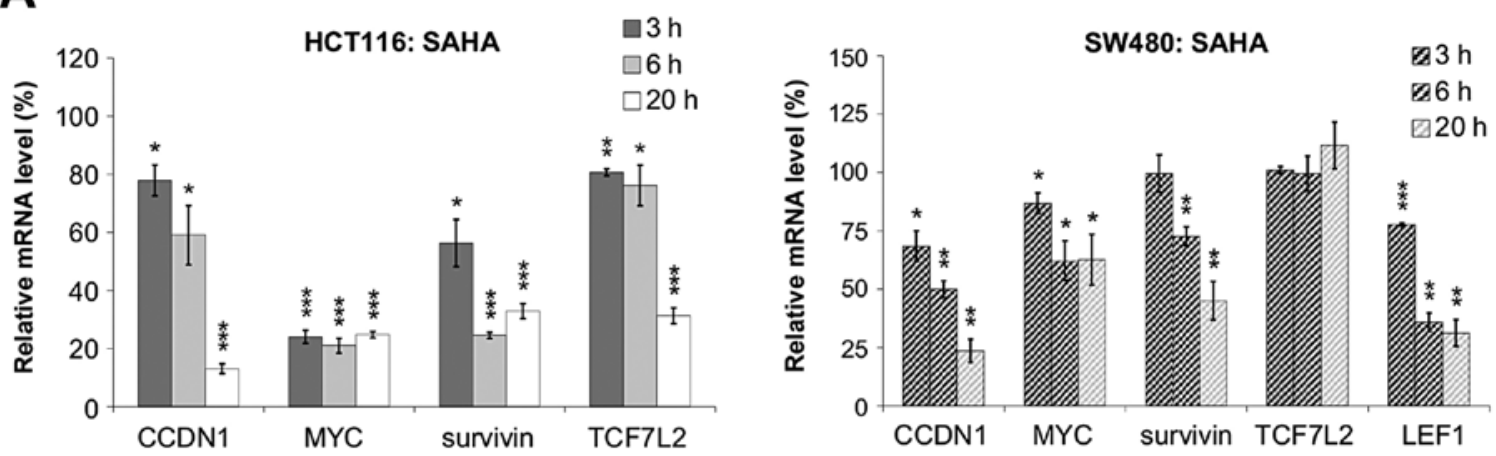

B
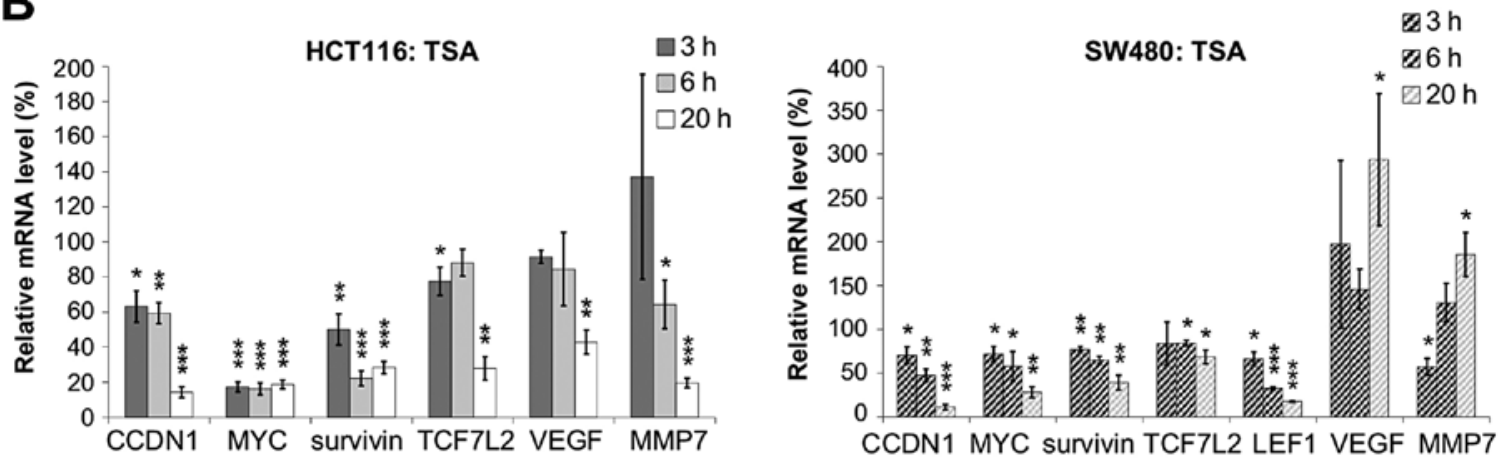

C
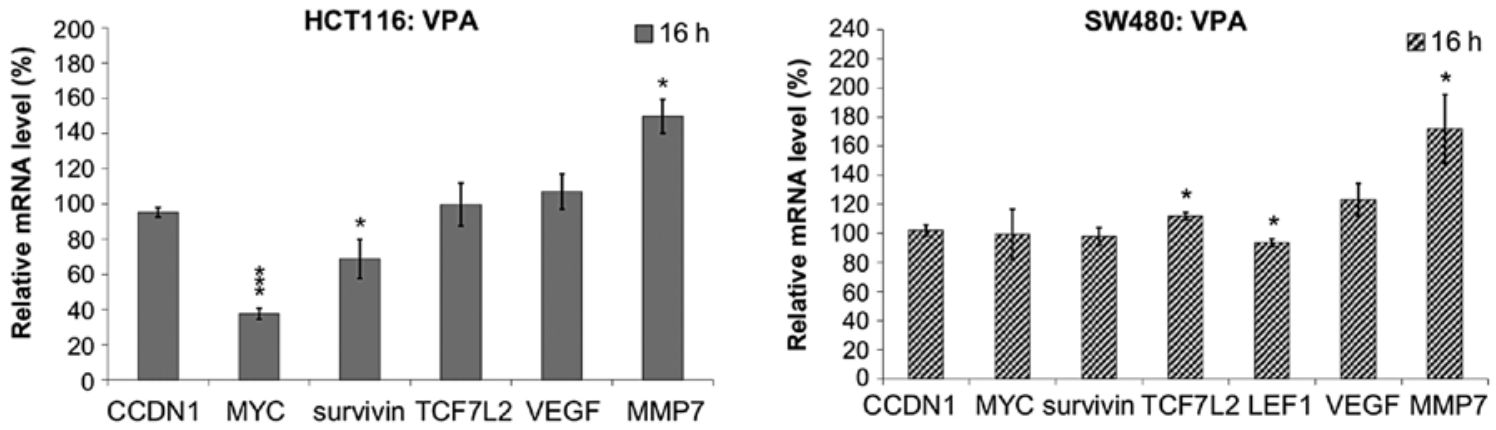

Figure 3. Effect of HDI treatment on Wnt target gene expression at different time points. Wnt target gene expression was analyzed by quantitative real-time PCR using the $\Delta \Delta \mathrm{Ct}$ method. Cells were treated with (A) $5 \mu \mathrm{M} \mathrm{SAHA}$, (B) $1 \mu \mathrm{M}$ TSA or (C) $1 \mathrm{mM}$ VPA for 3,6 or 16-20 h. Results are means of three independent experiments. The diagrams show the relative expression of the genes CCND1, MYC, survivin, TCF7L2, LEF1, VEGF and MMP7 in the cell lines HCT116 and SW480 in comparison to vehicle-treated cells (100\% relative expression). Wnt target gene expression of VPA treated cells was determined after $16 \mathrm{~h}$. The $V E G F$ and $M M P 7$ expression was analyzed after TSA and VPA treatment. Significant results are indicated by ${ }^{*} \mathrm{p}<0.05 ;{ }^{* *} \mathrm{p}<0.01$ or ${ }^{* * *} \mathrm{p}<0.001$.
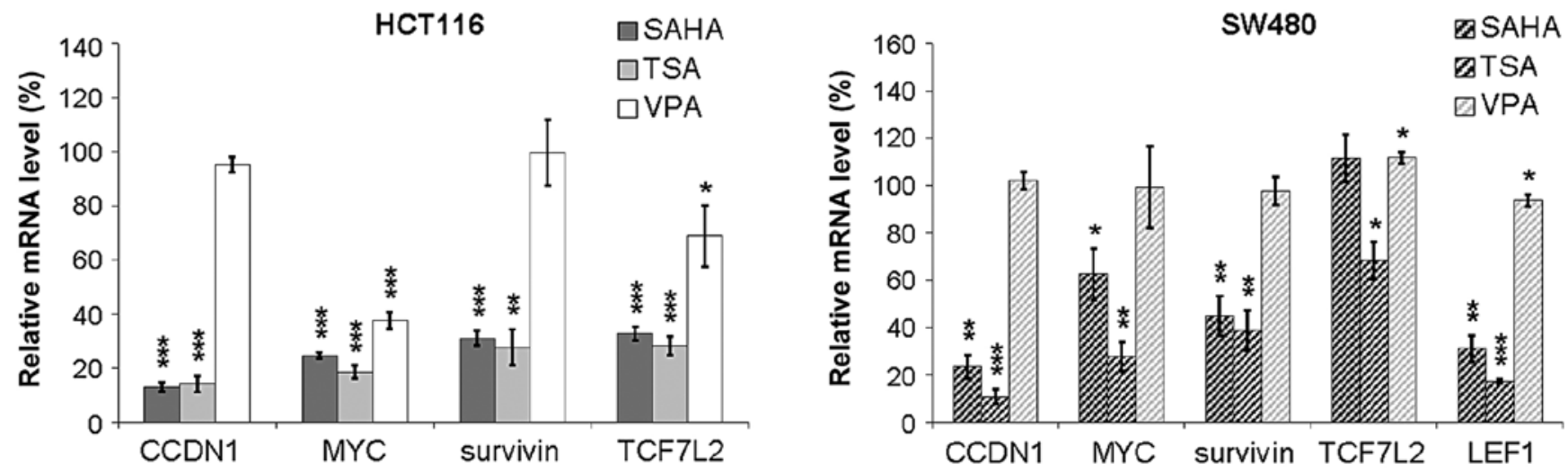

Figure 4. Effect of HDI treatment on Wnt target gene expression. Wnt target gene expression was analyzed by quantitative real-time PCR after treatment with $5 \mu \mathrm{M}$ SAHA, $1 \mu \mathrm{M}$ TSA or $1 \mathrm{mM}$ VPA for 16 to $20 \mathrm{~h}$. Results are means of three independent experiments. The relative expression of the genes $C C N D 1, M Y C$, survivin, TCF7L2 and LEF1 are shown for the cell lines HCT116 and SW480. Significant results are indicated by ${ }^{*} \mathrm{p}<0.05 ;{ }^{* *} \mathrm{p}<0.01$ or ${ }^{* * * *} \mathrm{p}<0.001$. 


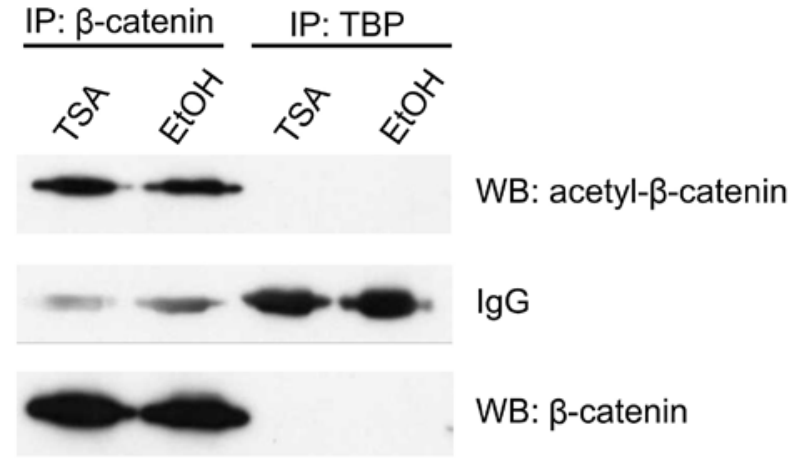

Figure 5. TSA treatment does not alter the acetylation of nuclear $\beta$-catenin in HCT116 cells. $\beta$-catenin was immunoprecipitated from nuclear lysates of HCT116 cells after $16 \mathrm{~h}$ treatment with TSA or EtOH. An anti-TBP antibody was used as unrelated internal control (TBP, tata-binding protein). The precipitate was probed with an anti-acetylated- $\beta$-catenin antibody and an anti- $\beta$-catenin antibody. IgG bands indicated equal loading of the different IP lysates.

of colon carcinoma cells and attenuation of Wnt signaling. In contrast, treatment with VPA, which inhibits only some HDAC classes, led to little, or no change, in the colon carci- noma cell lines. We reasoned that the different effects of the HDIs might be caused by the differences in their HDAC inhibition profiles. Therefore, we carried out siRNA experiments to analyze the effects of the knockdown of HDACs 6,10 and 11 , which are inhibited by hydroxamic acids but not by VPA, on Wnt signaling and colon cancer cell proliferation.

We controlled for a proper gene knockdown by measuring the mRNA expression level of the three HDACs 24 and $48 \mathrm{~h}$ after siRNA transfection. All three HDAC siRNAs reduced the respective HDAC expression to at least $40 \%$ after $48 \mathrm{~h}$ treatment. By using MTT, BrdU, apoptosis assay and flow cytometric analysis, we found that none of the analyzed HDAC siRNAs induced any significant changes in proliferation or apoptosis of HCT116 and SW480 cells. This indicated that the knockdown of a single HDAC is not sufficient to induce major changes in cell proliferation of the analyzed colon carcinoma cell lines.

Knockdown of HDAC 6,10 and 11 affects Wnt target gene expression. Next we examined whether siRNA mediated HDAC knockdown had an effect on Wnt target gene expression. Therefore, we performed quantitative real-time PCR of
A

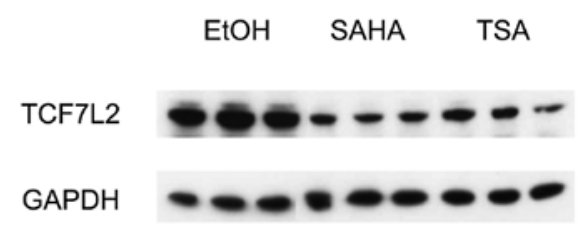

C

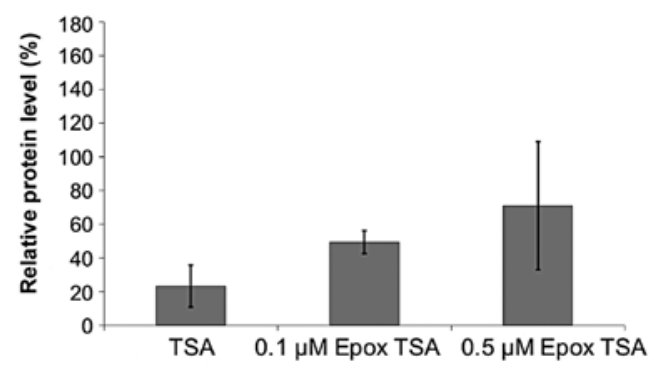

$\mathbf{E}$

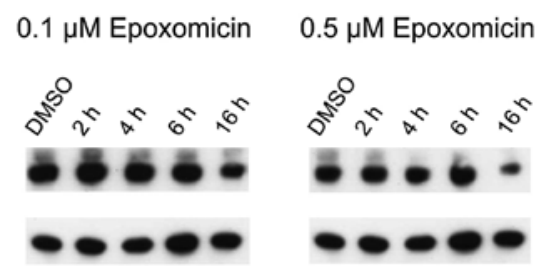

B

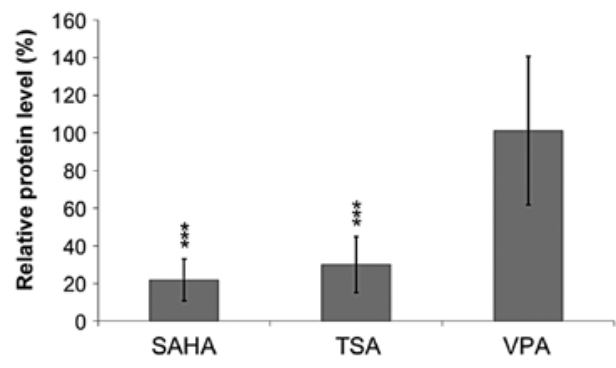

D

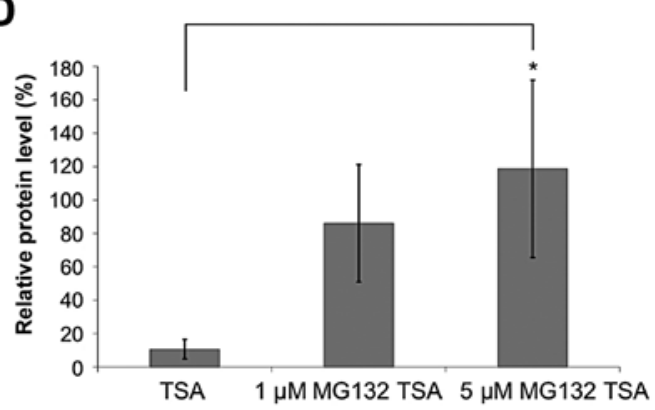

$1 \mu \mathrm{M} M \mathrm{MG} 132$

$5 \mu \mathrm{M} M \mathrm{MG} 132$

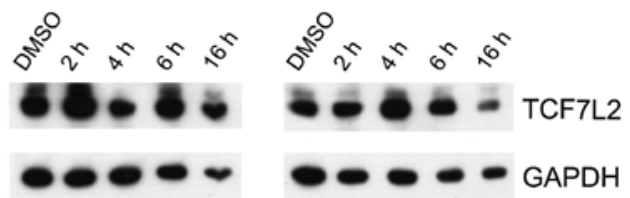

Figure 6. Analysis of TCF7L2 protein level revealing proteasome-dependent degradation after SAHA or TSA treatment. Diagrams show the densitometric analysis of western blots probed with TCF7L2 and GAPDH antibodies prepared from total lysates of HDI- and vehicle-treated HCT116 cells. (A) Representative images of the TCF7L2 and GAPDH western blot after $20 \mathrm{~h} \mathrm{SAHA}$ and TSA treatment. (B) Densitometric analysis of TCF7L2 after $20 \mathrm{~h}$ treatment with $5 \mu \mathrm{M}$ SAHA and $1 \mu \mathrm{M}$ TSA in HCT116 cells. (C) Densitometric analysis of TCF7L2 protein after $16 \mathrm{~h}$ TSA and $6 \mathrm{~h}$ epoxomicin or (D) after $16 \mathrm{~h}$ TSA and $4 \mathrm{~h}$ MG132 treatment in HCT116. (E) Western blot analysis of TCF7L2 and GAPDH protein after treatment of HCT116 with different concentrations of the proteasome inhibitors, epoxomicin and MG132 alone shows no substantial regulation after 2 to $6 \mathrm{~h}$ treatment. Significant results are indicated by ${ }^{*} \mathrm{p}<0.05 ;{ }^{* *} \mathrm{p}<0.01$ or ${ }^{* * * *} \mathrm{p}<0.001$. 
HCT116

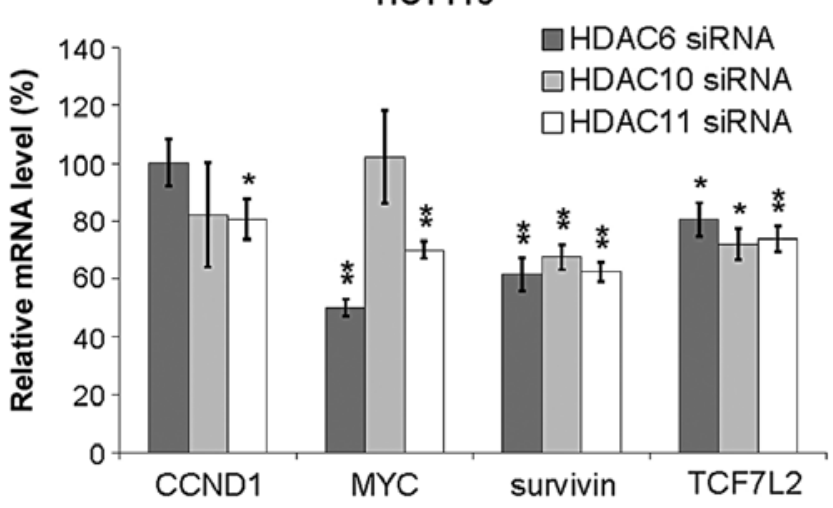

SW480

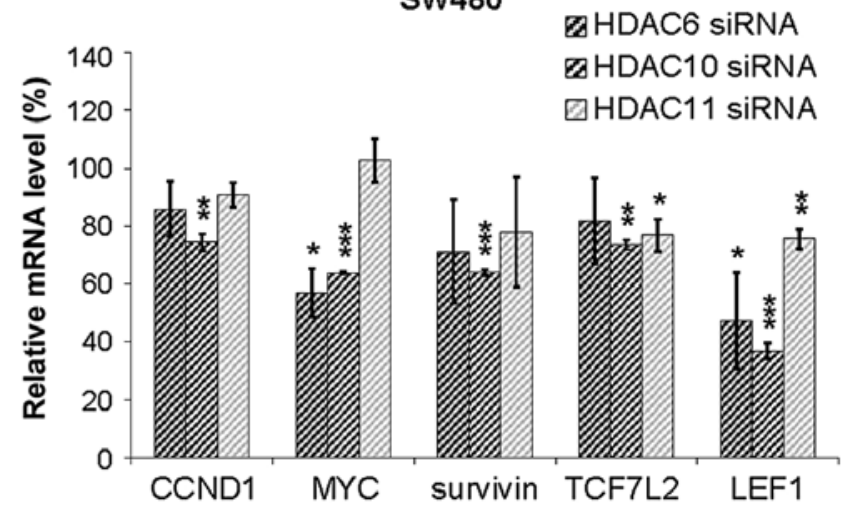

Figure 7. Effect of HDAC knockdown on Wnt target gene expression. Wnt target gene expression was determined $48 \mathrm{~h}$ after $H D A C$ siRNA transfection in HCT116 and SW480 cells. Results are means of three independent experiments. The relative expression levels of the genes $C C N D 1, M Y C$, survivin, TCF7L2 and $L E F 1$ are shown in comparison to the levels in cells transfected with non-targeting siRNA (100\% relative expression). Significant results are indicated by ${ }^{*} \mathrm{p}<0.05 ;{ }^{* *} \mathrm{p}<0.01$ or ${ }^{* * * *} \mathrm{p}<0.001$.

A

HDAC6 HDAC10 HDAC11 Non-target TCF7L2 GAPDH

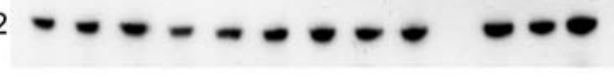

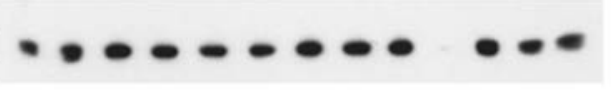

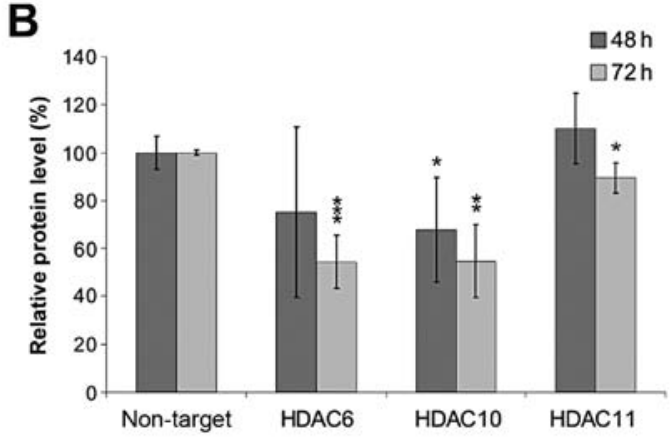

Figure 8. Analysis of TCF7L2 protein level upon HDAC knockdown. (A) Representative images of the TCF7L2 and GAPDH western blot analysis $72 \mathrm{~h}$ after siRNA transfection. (B) Densitometric analysis of the TCF7L2 western blots prepared from total lysates of HDAC and non-targeting siRNA transfected HCT116 cells. The TCF7L2 protein was significantly reduced $72 \mathrm{~h}$ after HDAC knockdown in HCT116. Significant results are indicated by ${ }^{*} \mathrm{p}<0.05 ;{ }^{* * *} \mathrm{p}<0.01$ or ${ }^{* * * *} \mathrm{p}<0.001$.

the endogenous Wnt target genes $C C D N 1, M Y C$, survivin, LEF1 and TCF7L2 $48 \mathrm{~h}$ after HDAC siRNA transfection.

In HCT116 cells, HDAC 6 or 10 knockdown reduced the expression of several Wnt target genes, whereas HDAC11 knockdown had an effect on all analyzed genes (Fig. 7). In SW480 cells, the transfection with HDAC10 siRNA led to a significant decrease in all analyzed Wnt target genes, while the siRNAs for HDAC 6 or 11 reduced only the expression of two of the five analyzed genes (MYC and LEF1 or LEF1 and TCF7L2, respectively) (Fig. 7).

Western blot analysis was performed to determine the effects of HDAC knockdown on the TCF7L2 protein level. The knockdown of HDACs 6 or 10 led to a reduction of the TCF7L2 protein level to approximately $50 \%$ of the initial protein level after $72 \mathrm{~h}(\mathrm{p}<0.001$ and $\mathrm{p}<0.01)$ (Fig. 8); while HDAC11 knockdown only slightly decreased the TCF7L2 protein level. Thus, HDACs 6 and 10 may be involved in the SAHA and TSA induced proteasomal degradation of TCF7L2 leading to the reduced expression of Wnt target genes.

\section{Discussion}

Through the regulation of a vast number of different target genes, active Wnt signaling is implicated in different aspects of tumorigenesis, including cell proliferation, apoptosis, angiogenesis and metastasis.

In this study, we observed the rapid induction of apoptotic colorectal carcinoma cell death and transcriptional down-regulation of Wnt target genes upon treatment with the hydroxamic acid HDIs SAHA and TSA. While SAHA and TSA rapidly induced apoptosis and cell death in colorectal carcinoma cells, we noted only slight effects upon VPA treatment. Likewise, APC mutant cells were relatively insensitive to VPA-induced apoptosis (20). However, VPA has been shown to be a potent inducer of apoptosis in various cancer models, including colorectal carcinoma cell lines (21), when cell viability was determined after 72-h treatment. In contrast, we found that after 24 and $48 \mathrm{~h}$ of treatment HCT116 and SW480 cells were much more sensitive to hydroxamic acid HDIs than to the fatty acid VPA.

The transcriptional regulation of Wnt target genes also displayed a differential mode of regulation upon HDI treatment. While TSA and SAHA downregulated Wnt target genes, VPA had no effects on the expression of most genes. In several different cellular contexts, the downregulation of single Wnt targets such as CCNDI or survivin upon HDI treatment has been described $(22,23)$. MMP7 expression was reduced by TSA and butyrate in chondrosarcoma cells (24). In addition, we found the two Wnt transcription factors TCF $2 L 2$ and $L E F 1$ 
to be downregulated by hydroxamic acids. However, as all HDIs induced histone hyperacetylation, the impact of HDIs on the Wnt pathway must be explained by a mechanism independent of histone hyperacetylation.

Recently, a growing number of non-histone targets of HDACs has been described, one of them being p53. Acetylation of p53 increases its binding affinity to target promoters and also its transactivation during DNA damage response (5). $\beta$-catenin also was shown to be a target of acetylation (25) which increases its affinity for TCF/LEF factors (26). However, we did not find a change in the $\beta$-catenin acetylation level upon TSA treatment.

Another non-histone target of HDACs is HSP90. HDI treatment disrupts HSP90 client protein interactions with subsequent proteasomal degradation of client proteins, e.g., Bcr-Abl (27). We analyzed if TCF7L2 might be regulated in a similar mode as HSP90 client proteins. Interestingly, TSA and SAHA induced depletion of TCF7L2 which could be attenuated by co-treatment with proteasome inhibitors. Given that TCF7L2 is the main transcription factor mediating Wnt target gene expression in colorectal carcinoma cells (28), TCF7L2 depletion might be the primary event in the observed Wnt target gene downregulation.

To elucidate the role of HDACs 6, 10 and 11, which are inhibited by hydroxamic acids but not by VPA (29), in Wnt signaling, we studied Wnt target gene expression and TCF7L2 protein level by siRNA experiments. Single knockdowns of HDACs 6, 10 and 11 reduced the expression of at least some of the analyzed Wnt target genes. In addition, knockdown of HDACs 6 or 10 induced a strong reduction in TCF7L2 protein level, while knockdown of HDAC11 induced a minor reduction. These results suggest that inhibition of HDACs 6 and 10 leads to the attenuation of Wnt signaling through the depletion of its main transcription factor TCF7L2. Interestingly, it was previously published that inhibition of HDACs 6 and 10 induces hyperacetylation of HSP90 leading to release and degradation of client proteins like VEGFR (30-32). Moreover, HSP90 inhibitors were shown to downregulate Wnt target gene expression (33). Taken together our data could indicate that TCF7L2 might be an HSP90 client protein, which has not been described yet.

To sum up the mode of HDI action on Wnt signaling, we suggest the following line of events: HDIs of the hydroxamate class inhibit the predominantly cytosolic HDACs 6 and 10 which results in hyperacetylation of a yet unidentified interaction partner of TCF7L2; thereby the release of TCF7L2 from its interaction partner is induced and TCF7L2 is subjected to proteasomal degradation. Concomitantly, Wnt target gene expression is downregulated and colorectal carcinoma cell death is induced. In numerous studies, the attenuation of Wnt signaling results in cancer cell death, e.g., TCF7L2 antisense RNA inhibits proliferation and in vivo tumor formation of liver cancer cell lines (34). The re-introduction of wild-type $A P C$ induces apoptosis in colorectal carcinoma cells (35). Likewise, a Wntl antibody or small molecule inhibitors of Wnt signaling stimulate apoptosis in different types of human cancer cells $(36,37)$. Other reports show that active Wnt signaling inhibits apoptosis in cancer cells (38). Thus, TCF7L2 depletion and attenuation of Wnt signaling renders cancer cells sensitive to apoptotic stimuli.

In conclusion, we describe the depletion of TCF7L2 by HDIs of the hydroxamate class, through the inhibition of HDAC6 and HDAC10, leading to an attenuation of Wnt signaling. TCF7L2 degradation might be the primary event in the observed Wnt target gene downregulation and apoptotic colorectal carcinoma cell death. These findings provide a molecular rationale for the use of HDIs against colorectal carcinomas with activated Wnt signaling.

\section{Acknowledgements}

S.G. was supported by a predoctorial grant of the Hans-Böckler Stiftung.

\section{References}

1. Insinga A, Monestiroli S, Ronzoni S, et al: Inhibitors of histone deacetylases induce tumor-selective apoptosis through activation of the death receptor pathway. Nat Med 11: 71-76, 2005.

2. Schrump DS: Cytotoxicity mediated by histone deacetylase inhibitors in cancer cells: mechanisms and potential clinical implications. Clin Cancer Res 15: 3947-3957, 2009.

3. Rada-Iglesias A, Enroth S, Ameur A, et al: Butyrate mediates decrease of histone acetylation centered on transcription start sites and down-regulation of associated genes. Genome Res 17: 708-719, 2007.

4. Hu J and Colburn NH: Histone deacetylase inhibition down-regulates cyclin D1 transcription by inhibiting nuclear factor-kappaB/p65 DNA binding. Mol Cancer Res 3: 100-109, 2005.

5. Luo J, Li M, Tang Y, Laszkowska M, Roeder RG and Gu W: Acetylation of p53 augments its site-specific DNA binding both in vitro and in vivo. Proc Natl Acad Sci USA 101: 2259-2264, 2004.

6. Jin YH, Jeon EJ, Li QL, et al: Transforming growth factorbeta stimulates p300-dependent RUNX3 acetylation, which inhibits ubiquitination-mediated degradation. J Biol Chem 279: 29409-29417, 2004.

7. Piekarz RL and Bates SE: Epigenetic modifiers: basic understanding and clinical development. Clin Cancer Res 15: 3918-3926, 2009.

8. Kim HJ and Bae SC: Histone deacetylase inhibitors: molecular mechanisms of action and clinical trials as anti-cancer drugs. Am J Transl Res 3: 166-179, 2011.

9. Kobayashi H, Tan EM and Fleming SE: Acetylation of histones associated with the p21WAF1/CIP1 gene by butyrate is not sufficient for p21WAF1/CIP1 gene transcription in human colorectal adenocarcinoma cells. Int J Cancer 109: 207-213, 2004.

10. Chen YX, Fang JY, Zhu HY, Lu R, Cheng ZH and Qiu DK: Histone acetylation regulates p21WAF1 expression in human colon cancer cell lines. World J Gastroenterol 10: 2643-2646, 2004.

11. Zhu P, Martin E, Mengwasser J, Schlag P, Janssen KP and Gottlicher M: Induction of HDAC2 expression upon loss of APC in colorectal tumorigenesis. Cancer Cell 5: 455-463, 2004.

12. Reya $\mathrm{T}$ and Clevers $\mathrm{H}$ : Wnt signalling in stem cells and cancer. Nature 434: 843-850, 2005.

13. Willert $\mathrm{K}$ and Jones KA: Wnt signaling: is the party in the nucleus? Genes Dev 20: 1394-1404, 2006.

14. Morin PJ, Sparks AB, Korinek V, et al: Activation of beta-catenin-Tcf signaling in colon cancer by mutations in beta-catenin or APC. Science 275: 1787-1790, 1997.

15. Lallemand F, Courilleau D, Sabbah M, Redeuilh G and Mester J: Direct inhibition of the expression of cyclin D1 gene by sodium butyrate. Biochem Biophys Res Commun 229: 163-169, 1996.

16. Kim MS, Kwon HJ, Lee YM, et al: Histone deacetylases induce angiogenesis by negative regulation of tumor suppressor genes. Nat Med 7: 437-443, 2001.

17. Bordonaro M, Lazarova DL, Augenlicht LH and Sartorelli AC: Cell type- and promoter-dependent modulation of the Wnt signaling pathway by sodium butyrate. Int J Cancer 97: 42-51, 2002.

18. Heruth DP, Zirnstein GW, Bradley JF and Rothberg PG: Sodium butyrate causes an increase in the block to transcriptional elongation in the c-myc gene in SW837 rectal carcinoma cells. J Biol Chem 268: 20466-20472, 1993. 
19. Varshavsky A, Bachmair A and Finley D: The N-end rule of selective protein turnover: mechanistic aspects and functional implications. Biochem Soc Trans 15: 815-816, 1987.

20. Huang $X$ and Guo B: Adenomatous polyposis coli determines sensitivity to histone deacetylase inhibitor-induced apoptosis in colon cancer cells. Cancer Res 66: 9245-9251, 2006.

21. Mologni L, Cleris L, Magistroni V, et al: Valproic acid enhances bosutinib cytotoxicity in colon cancer cells. Int J Cancer 124: 1990-1996, 2009.

22. Jin JS, Tsao TJ, Sun PC, Yu CT and Tzao C: SAHA inhibits the growth of colon tumors by decreasing histone deacetylase and the expression of cyclin D1 and survivin. Pathol Oncol Res 18: 713-720, 2012.

23. Hsu YF, Sheu JR, Lin CH, et al: Trichostatin A and sirtinol suppressed survivin expression through AMPK and p38MAPK in HT29 colon cancer cells. Biochim Biophys Acta 1820: 104-115, 2012

24. Young DA, Lakey RL, Pennington CJ, et al: Histone deacetylase inhibitors modulate metalloproteinase gene expression in chondrocytes and block cartilage resorption. Arthritis Res Ther 7: R503-R512, 2005.

25. Wolf D, Rodova M, Miska EA, Calvet JP and Kouzarides T: Acetylation of beta-catenin by CREB-binding protein (CBP). J Biol Chem 277: 25562-25567, 2002.

26. Levy L, Wei Y, Labalette C, et al: Acetylation of beta-catenin by p300 regulates beta-catenin-Tcf4 interaction. Mol Cell Biol 24: 3404-3414, 2004.

27. Nimmanapalli R, Fuino L, Bali P, et al: Histone deacetylase inhibitor LAQ824 both lowers expression and promotes proteasomal degradation of Bcr-Abl and induces apoptosis of imatinib mesylate-sensitive or -refractory chronic myelogenous leukemia-blast crisis cells. Cancer Res 63: 5126-5135, 2003.

28. Korinek V, Barker N, Morin PJ, et al: Constitutive transcriptional activation by a beta-catenin-Tcf complex in $\mathrm{APC}^{-/}$colon carcinoma. Science 275: 1784-1787, 1997.
29. Bolden JE, Peart MJ, Johnstone RW: Anticancer activities of histone deacetylase inhibitors. Nat Rev Drug Discov 5: 769-784, 2006.

30. Bali P, Pranpat M, Bradner J, Balasis M, et al: Inhibition of histone deacetylase 6 acetylates and disrupts the chaperone function of heat shock protein 90: a novel basis for antileukemia activity of histone deacetylase inhibitors. J Biol Chem 280: 26729-26734, 2005

31. Park JH, Kim SH, Choi MC, et al: Class II histone deacetylases play pivotal roles in heat shock protein 90 -mediated proteasomal degradation of vascular endothelial growth factor receptors. Biochem Biophys Res Commun 368: 318-322, 2008.

32. New M, Olzscha $\mathrm{H}$ and La Thongue NB: HDAC inhibitor-based therapies: Can we interpret the code? Mol Oncol 6: 637-656, 2012.

33. Kurashina R, Ohyashiki JH, Kobayashi C, et al: Anti-proliferative activity of heat shock protein (Hsp) 90 inhibitors via beta-catenin/TCF7L2 pathway in adult T cell leukemia cells. Cancer Lett 284: 62-70, 2009.

34. Jiang Y, Zhou XD, Liu YK, et al: Antisense Tcf inhibits the neoplastic growth of liver cancer cells. J Cancer Res Clin Oncol 130: 671-678, 2004.

35. Morin PJ, Vogelstein B and Kinzler KW: Apoptosis and APC in colorectal tumorigenesis. Proc Natl Acad Sci USA 93: 7950-7954, 1996.

36. He B, You L, Uematsu K, et al: A monoclonal antibody against Wnt-1 induces apoptosis in human cancer cells. Neoplasia 6: 7-14, 2004.

37. Minke KS, Staib P, Puetter A, et al: Small molecule inhibitors of WNT signaling effectively induce apoptosis in acute myeloid leukemia cells. Eur J Haematol 82: 165-175, 2009.

38. Chen S, Guttridge DC, You Z, et al: Wnt-1 signaling inhibits apoptosis by activating beta-catenin/T cell factor-mediated transcription. J Cell Biol 152: 87-96, 2001. 